\title{
Aspectos relevantes sobre la estadística de deficiencias de la nutrición*
}

L a Dirección General de Estadística e Informática presenta algunos de los cuadros estadísticos más importantes acerca de los daños a la salud, morbilidad y mortalidad, que tienen relación con las deficiencias del estado nutricional.

Para la mortalidad fueron seleccionadas dos fuentes: la del Instituto Nacional de Estadística, Geografía e Informática, que genera la estadística de defunciones del país, y la del Boletín de Información Estadística, la cual integra las defunciones ocurridas en hospitales del Sistema Nacional de Salud (SNS).

A partir de la primera fuente, los datos han sido procesados a fin de obtener la tendencia para el total de defunciones por sexo entre 1990 y 1996; se presenta además un cuadro comparativo por grupos de edad y sexo para 1996, otro para las causas relacionadas con las deficiencias de la nutrición y con el desglose que da la lista detallada de la Clasificación Internacional de Enfermedades y, finalmente, dos cuadros por entidad federativa con el detalle del número de defunciones por sexo y grupos de edad, así como de las muertes por dichas deficiencias según los principales grupos de causas.
Por otra parte, la mortalidad que se registra en los hospitales del SNS permite obtener otro punto de vista para el análisis de este tipo de causas, que son motivo de atención en el segundo y el tercer niveles. Los cuadros muestran las defunciones por tipo de institución y las tasas de letalidad entre 1991 y 1996, años para los cuales se dispone de información con este detalle.

En relación con la mortalidad, es importante señalar que las deficiencias nutricionales pocas veces se consideran en la selección de la causa básica de defunción, debido a que en muchos casos se comportan como causas intervinientes, contribuyentes o asociadas con la causa que, a juicio de los médicos, inició la cadena de acontecimientos que llevaron a la muerte. Con la estadística tradicional de causa única (básica) la importancia de dichas deficiencias puede verse reducida, pues sólo se hace evidente cuando el estudio de la mortalidad se aborda bajo el enfoque de causas múltiples, donde se toman en cuenta todas las anotadas en los certificados.

En cuanto a la morbilidad, se incluyen los resultados de los censos de talla practicados en 1993 y 1994 por el Sistema para el Desarrollo In- tegral de la Familia en niños de primer grado de primaria, en los cuales se presentó un déficit equivalente a dos desviaciones estándar del valor medio esperado para la talla. Con este indicador se hacen evidentes las grandes diferencias entre los estados según las diversas condiciones de desarrollo.

Para la morbilidad hospitalaria se muestra un tabulado con los egresos notificados, durante el periodo 1991-1996, por las instituciones del SNS, para los casos en los que las deficiencias nutricionales fueron la afección principal.

Del Sistema de Información en Salud para Población Abierta, se presentan las consultas impartidas en escala nacional a los menores de cinco años en las unidades de la Secretaría de Salud (SSA), según el grado nutricional de los niños, durante el periodo 1990-1996; para este último año, la información se presenta por entidad federativa. Aunque las cifras no corresponden a personas sino a consultas, este indicador mensual permite tener una idea de la condición nutricional de los niños que son atendidos por la SSA.

\footnotetext{
* Sección preparada por la Dirección General de Estadística e Informática de la Secretaría de Salud, México.
} 


\section{Cuadro I}

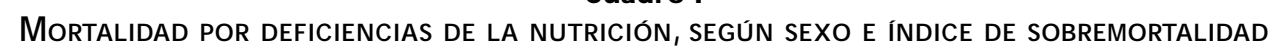
Estados Unidos Mexicanos, 1996

\begin{tabular}{|c|c|c|c|c|c|c|c|c|}
\hline Años & \multicolumn{2}{|c|}{$\begin{array}{ccccc} & 0 & t & a & 1\end{array}$} & \multicolumn{2}{|c|}{$\frac{H \circ m b r \text { e } s}{\text { Defunciones }}$} & $\frac{M u j}{\text { Defunciones }}$ & $\frac{s}{\text { Tasa }}$ & $\begin{array}{c}\text { NE } \\
\text { Defunciones }\end{array}$ & ISM \\
\hline 1990 & 11788 & 14.1 & 6015 & 14.5 & 5738 & 13.7 & 35 & 104.8 \\
\hline 1991 & 11022 & 12.9 & 5584 & 13.2 & 5425 & 12.7 & 13 & 102.9 \\
\hline 1992 & 10238 & 11.8 & 4993 & 11.6 & 5241 & 12.0 & 4 & 95.3 \\
\hline 1993 & 10011 & 11.3 & 5085 & 11.6 & 4921 & 11.0 & 5 & 103.3 \\
\hline 1994 & 9585 & 10.6 & 4675 & 10.5 & 4908 & 10.8 & 2 & 95.3 \\
\hline 1995 & 10162 & 11.1 & 4955 & 10.9 & 5206 & 11.3 & 1 & 95.2 \\
\hline 1996 & 10269 & 11.0 & 5015 & 10.9 & 5254 & 11.2 & 0 & 95.5 \\
\hline
\end{tabular}

ISM = Indice de sobremortalidad masculina en porcentaje, es decir, número de defunciones masculinas por 100 femeninas $\mathrm{NE}=$ no especificado

Fuente: Instituto N acional de Estadística, Geografía e Informática; Dirección General de Estadística e Informática, Secretaría de Salud

\section{Cuadro II \\ MORTALIDAD POR DEFICIENCIAS DE LA NUTRICIÓN, SEGÚN GRUPO DE EDAD Y SEXO Estados Unidos MeXicanos, 1996}

\begin{tabular}{|c|c|c|c|c|c|c|c|}
\hline \multirow{2}{*}{$\begin{array}{l}\text { Grupo de } \\
\text { edad (años) }\end{array}$} & \multicolumn{2}{|c|}{ Total } & \multicolumn{2}{|c|}{ Hombres } & \multicolumn{2}{|c|}{ Mujeres } & \multirow[b]{2}{*}{ ISM * } \\
\hline & No. & Tasa $^{\ddagger}$ & No. & Tasa $^{\ddagger}$ & No. & Tasa $^{\ddagger}$ & \\
\hline Total & 10269 & 11.0 & 5015 & 10.9 & 5254 & 11.2 & 95.5 \\
\hline$<1$ & 1326 & 1.4 & 742 & 1.6 & 584 & 1.2 & 127.1 \\
\hline $1-4$ & 798 & 0.9 & 401 & 0.9 & 397 & 0.8 & 101.0 \\
\hline $5-10$ & 222 & 0.2 & 111 & 0.2 & 111 & 0.2 & 100.0 \\
\hline $15-24$ & 160 & 0.2 & 82 & 0.2 & 78 & 0.2 & 105.1 \\
\hline $25-34$ & 189 & 0.2 & 119 & 0.3 & 70 & 0.1 & 170.0 \\
\hline $35-44$ & 252 & 0.3 & 153 & 0.3 & 99 & 0.2 & 154.5 \\
\hline $45-54$ & 296 & 0.3 & 174 & 0.4 & 122 & 0.3 & 142.6 \\
\hline $55-64$ & 505 & 0.5 & 279 & 0.6 & 226 & 0.5 & 123.5 \\
\hline $65 \mathrm{y}+$ & 6452 & 6.9 & 2909 & 6.3 & 3543 & 7.5 & 82.1 \\
\hline$\overline{N E}$ & 69 & 0.1 & 45 & 0.1 & 24 & 0.1 & 187.5 \\
\hline
\end{tabular}

\footnotetext{
* ISM = Indice de sobremortalidad masculina en porcentaje, es decir, número de defunciones masculinas por 100 femeninas

₹ Tasa por 100000 habitantes con base en las estimaciones de población del Consejo Nacional de Población

$\mathrm{NE}=$ no especificado
}

Fuente: Instituto N acional de Estadística, Geografía e Informática; Dirección General de Estadística e Informática, Secretaría de Salud 


\section{Cuadro III \\ MoRTALIDAd POR Deficiencias de LA NUTRICIÓN A LiSTA DETALLADA SEGÚn SEXo Estados Unidos Mexicanos, 1996}

\begin{tabular}{|c|c|c|c|c|c|c|c|c|}
\hline & & To & & $\mathrm{Hom}$ & bres & Muj & eres & \\
\hline Descripción & Causa & No. & Tasa $^{\ddagger}$ & No. & Tasa $^{\ddagger}$ & No. & Tasa $^{\ddagger}$ & ISM* \\
\hline Total & $260-269$ & 10269 & 11.02 & 5015 & 10.86 & 5254 & 11.18 & 95.5 \\
\hline Kwashiorkor (edema nutricional con despigmentación de la piel y del pelo) & 260 & 116 & 0.12 & 55 & 0.12 & 61 & 0.13 & 90.2 \\
\hline Marasmo nutricional & 261 & 845 & 0.91 & 401 & 0.87 & 444 & 0.94 & 90.3 \\
\hline 0 tra desnutrición proteinocalórica & $262-263$ & 9238 & 9.91 & 4526 & 9.80 & 4712 & 10.03 & 96.1 \\
\hline 0 tra desnutrición proteinocalórica grave & $262 X$ & 3465 & 3.72 & 1735 & 3.76 & 1730 & 3.68 & 100.3 \\
\hline Desnutrición de grado moderado & 2630 & 1095 & 1.18 & 549 & 1.19 & 546 & 1.16 & 100.5 \\
\hline Desnutrición de grado leve & 2631 & 143 & 0.15 & 70 & 0.15 & 73 & 0.16 & 95.9 \\
\hline 0 tras formas de desnutrición proteinocalórica & 2638 & 7 & 0.01 & 6 & 0.01 & 1 & 0.00 & 600.0 \\
\hline 0 tras formas de desnutrición proteinocalórica sin especificación & 2639 & 4528 & 4.86 & 2166 & 4.69 & 2362 & 5.03 & 91.7 \\
\hline Avitaminosis & $264-269$ & 70 & 0.08 & 33 & 0.07 & 37 & 0.08 & 89.2 \\
\hline Deficiencia de vitamina $A$, con xerosis de la córnea & 2642 & 1 & 0.00 & 0 & 0.00 & 1 & 0.00 & 0.0 \\
\hline D eficiencia de vitamina A, sin especificación & 2649 & 1 & 0.00 & 0 & 0.00 & 1 & 0.00 & 0.0 \\
\hline 0 tras manifestaciones de la deficiencia de tiamina y las no especificadas & 2651 & 4 & 0.00 & 3 & 0.01 & 1 & 0.00 & 300.0 \\
\hline Pelagra (deficiencia de ácido nicotínico) & 2652 & 6 & 0.01 & 2 & 0.00 & 4 & 0.01 & 50.0 \\
\hline 0 tras deficiencias del complejo B & 2662 & 1 & 0.00 & 1 & 0.00 & 0 & 0.00 & -- \\
\hline Raquitismo activo & 2680 & 5 & 0.01 & 5 & 0.01 & 0 & 0.00 & -- \\
\hline Efectos tardíos del raquitismo & 2681 & 2 & 0.00 & 1 & 0.00 & 1 & 0.00 & 100.0 \\
\hline 0 steomalacia & 2682 & 4 & 0.00 & 2 & 0.00 & 2 & 0.00 & 100.0 \\
\hline D eficiencia de vitamina no especificada & 2692 & 44 & 0.05 & 19 & 0.04 & 25 & 0.05 & 76.0 \\
\hline 0 tras deficiencias nutricionales, sin especificación & 2699 & 2 & 0.00 & 0 & 0.00 & 2 & 0.00 & 0.0 \\
\hline
\end{tabular}

* ISM = Indice de sobremortalidad masculina en porcentaje, es decir, número de defunciones masculinas por 100 femeninas

₹ Tasa por 100000 habitantes con base en las estimaciones de población del Consejo $\mathrm{N}$ acional de Población

Fuente: Instituto N acional de Estadística, Geografía e Informática; Dirección General de Estadística e Informática, Secretaría de Salud 


\section{Cuadro IV}

\section{MORTALIDAD POR DEFICIENCIAS DE LA NUTRICIÓN SEGÚN ENTIDAD FEDERATIVA, SEXO Y GRUPO DE EDAD Estados Unidos Mexicanos, 1996}

\begin{tabular}{|c|c|c|c|c|c|c|c|c|c|c|c|c|c|}
\hline & & $\mathrm{Se}$ & $\times 0$ & & & & & e e & a d & a ñ 0 & & & \\
\hline itidad federativa & Total & Hombres & M ujeres & $<1$ & $1-4$ & $5-10$ & $15-24$ & $25-34$ & $35-44$ & $45-54$ & $55-64$ & $65 y+$ & NE \\
\hline tados Unidos Mexicanos & 0269 & 5015 & 5254 & 1326 & 798 & 222 & 160 & 189 & 252 & 296 & 505 & 6452 & 69 \\
\hline
\end{tabular}

\begin{tabular}{|c|c|c|c|c|c|c|c|c|c|c|c|c|}
\hline Aguascalientes & 91 & 43 & 48 & 13 & 1 & 2 & 1 & 1 & 0 & 2 & 5 & 66 \\
\hline Baja California & 113 & 58 & 55 & 12 & 5 & 2 & 0 & 1 & 3 & 5 & 5 & 75 \\
\hline Baja California Sur & 37 & 19 & 18 & 3 & 4 & 0 & 2 & 0 & 0 & 2 & 1 & 25 \\
\hline Campeche & 56 & 26 & 30 & 4 & 2 & 0 & 0 & 2 & 0 & 1 & 1 & 46 \\
\hline Coahuila & 173 & 84 & 89 & 8 & 2 & 0 & 4 & 2 & 1 & 5 & 13 & 137 \\
\hline Colima & 59 & 34 & 25 & 4 & 4 & 1 & 0 & 2 & 1 & 1 & 1 & 44 \\
\hline Chiapas & 402 & 186 & 216 & 62 & 52 & 25 & 5 & 12 & 12 & 6 & 30 & 192 \\
\hline Chihuahua & 282 & 157 & 125 & 35 & 35 & 8 & 5 & 8 & 4 & 7 & 13 & 164 \\
\hline Distrito Federal & 487 & 230 & 257 & 54 & 4 & 8 & 11 & 20 & 12 & 13 & 28 & 336 \\
\hline Durango & 77 & 39 & 38 & 3 & 1 & 1 & 0 & 1 & 1 & 6 & 9 & 55 \\
\hline Guanajuato & 765 & 348 & 417 & 130 & 52 & 8 & 8 & 7 & 8 & 20 & 22 & 501 \\
\hline Guerrero & 303 & 154 & 149 & 29 & 43 & 5 & 13 & 8 & 9 & 12 & 12 & 172 \\
\hline Hidalgo & 384 & 170 & 214 & 31 & 28 & 7 & 2 & 8 & 17 & 18 & 22 & 247 \\
\hline Jalisco & 602 & 284 & 318 & 62 & 22 & 7 & 8 & 6 & 20 & 17 & 27 & 432 \\
\hline México & 1002 & 505 & 497 & 230 & 82 & 33 & 9 & 21 & 22 & 33 & 56 & 511 \\
\hline Michoacán & 399 & 193 & 206 & 37 & 22 & 6 & 2 & 6 & 8 & 12 & 15 & 291 \\
\hline Morelos & 169 & 83 & 86 & 15 & 5 & 3 & 2 & 4 & 7 & 6 & 9 & 115 \\
\hline $\mathrm{N}$ ayarit & 63 & 28 & 35 & 5 & 7 & 0 & 2 & 1 & 2 & 2 & 2 & 41 \\
\hline N uevo León & 158 & 76 & 82 & 12 & 6 & 2 & 1 & 5 & 3 & 6 & 4 & 117 \\
\hline 0 axaca & 967 & 445 & 522 & 94 & 116 & 22 & 12 & 14 & 30 & 32 & 54 & 592 \\
\hline Puebla & 1068 & 493 & 575 & 185 & 119 & 32 & 24 & 13 & 31 & 28 & 47 & 587 \\
\hline Q uerétaro & 193 & 103 & 90 & 19 & 18 & 1 & 0 & 2 & 4 & 2 & 8 & 138 \\
\hline Q uintana Roo & 35 & 20 & 15 & 6 & 4 & 2 & 0 & 1 & 0 & 2 & 5 & 14 \\
\hline San Luis Potosí & 264 & 118 & 146 & 28 & 28 & 4 & 7 & 4 & 5 & 2 & 13 & 171 \\
\hline Sinaloa & 132 & 75 & 57 & 13 & 4 & 3 & 0 & 3 & 5 & 3 & 5 & 95 \\
\hline Sonora & 173 & 104 & 69 & 16 & 8 & 1 & 2 & 7 & 6 & 3 & 12 & 117 \\
\hline Tabasco & 175 & 84 & 91 & 34 & 13 & 3 & 5 & 3 & 6 & 7 & 4 & 99 \\
\hline Tamaulipas & 215 & 117 & 98 & 23 & 6 & 1 & 2 & 2 & 4 & 5 & 13 & 159 \\
\hline Tlaxcala & 136 & 63 & 73 & 29 & 7 & 2 & 1 & 2 & 0 & 0 & 4 & 91 \\
\hline Veracruz & 876 & 451 & 425 & 108 & 80 & 27 & 24 & 18 & 22 & 32 & 48 & 509 \\
\hline Yucatán & 238 & 134 & 104 & 8 & 16 & 4 & 5 & 4 & 6 & 5 & 8 & 175 \\
\hline Zacatecas & 170 & 90 & 80 & 13 & 2 & 1 & 3 & 1 & 3 & 1 & 8 & 136 \\
\hline Extranjero & 5 & 1 & 4 & 1 & 0 & 1 & 0 & 0 & 0 & 0 & 1 & 2 \\
\hline
\end{tabular}

$N E=$ no especificado

Fuente: Instituto N acional de Estadística, Geografía e Informática; Dirección General de Estadística e Informática, Secretaría de Salud 


\section{Cuadro V}

\section{MORTALIDAD POR DEFICIENCIAS DE LA NUTRICIÓN A LISTA BÁSICA SEGÚN ENTIDAD FEDERATIVA Estados Unidos Mexicanos, 1996}

\begin{tabular}{|c|c|c|c|c|c|}
\hline Entidad federativa & Total & $\begin{array}{l}\text { Kwashiorkor (edema nutricional } \\
\text { con despigmentación de la piel y del pelo) }\end{array}$ & Marasmo nutricional & $\begin{array}{l}\text { Otra desnutrición } \\
\text { proteinocalórica }\end{array}$ & Avitaminosis \\
\hline Estados Unidos Mexicanos & 10269 & 116 & 845 & 9238 & 70 \\
\hline Aguascalientes & 91 & 0 & 1 & 88 & 2 \\
\hline Baja California & 113 & 0 & 1 & 110 & 2 \\
\hline Baja California Sur & 37 & 0 & 0 & 37 & 0 \\
\hline Campeche & 56 & 1 & 0 & 55 & 0 \\
\hline Coahuila & 173 & 2 & 22 & 149 & 0 \\
\hline Colima & 59 & 1 & 7 & 51 & 0 \\
\hline Chiapas & 402 & 8 & 80 & 310 & 4 \\
\hline Chihuahua & 282 & 1 & 1 & 278 & 2 \\
\hline Distrito Federal & 487 & 0 & 75 & 409 & 3 \\
\hline Durango & 77 & 3 & 0 & 74 & 0 \\
\hline Guanajuato & 765 & 13 & 8 & 743 & 1 \\
\hline Guerrero & 303 & 3 & 4 & 294 & 2 \\
\hline Hidalgo & 384 & 0 & 52 & 331 & 1 \\
\hline Jalisco & 602 & 5 & 48 & 544 & 5 \\
\hline México & 1002 & 6 & 13 & 976 & 7 \\
\hline Michoacán & 399 & 2 & 21 & 370 & 6 \\
\hline Morelos & 169 & 1 & 0 & 166 & 2 \\
\hline $\mathrm{N}$ ayarit & 63 & 1 & 11 & 51 & 0 \\
\hline Nuevo León & 158 & 1 & 36 & 121 & 0 \\
\hline 0 axaca & 967 & 8 & 174 & 773 & 12 \\
\hline Puebla & 1068 & 2 & 164 & 896 & 6 \\
\hline Q uerétaro & 193 & 2 & 0 & 190 & 1 \\
\hline Q uintana Roo & 35 & 3 & 1 & 30 & 1 \\
\hline San Luis Potosí & 264 & 3 & 1 & 258 & 2 \\
\hline Sinaloa & 132 & 4 & 0 & 128 & 0 \\
\hline Sonora & 173 & 4 & 0 & 168 & 1 \\
\hline Tabasco & 175 & 3 & 26 & 146 & 0 \\
\hline Tamaulipas & 215 & 2 & 26 & 187 & 0 \\
\hline Tlaxcala & 136 & 0 & 12 & 124 & 0 \\
\hline Veracruz & 876 & 14 & 58 & 803 & 1 \\
\hline Yucatán & 238 & 19 & 2 & 209 & 8 \\
\hline Zacatecas & 170 & 4 & 1 & 164 & 1 \\
\hline Extranjero & 5 & 0 & 0 & 5 & 0 \\
\hline
\end{tabular}

Fuente: Instituto N acional de Estadística, Geografía e Informática; Dirección General de Estadística e Informática, Secretaría de Salud 


\title{
Cuadro VI
}

\section{MoRTALIDAd hOSPITALARIA POR DEFICIENCIAS DE LA NUTRICIÓN, POBLACIÓN ABIERTA Estados Unidos Mexicanos, 1991-1996}

\begin{tabular}{|c|c|c|c|c|c|c|c|c|c|c|c|c|c|c|c|c|c|}
\hline \multirow[b]{2}{*}{ Años } & \multicolumn{2}{|c|}{ Tot a l } & \multicolumn{12}{|c|}{$P \quad 0 \quad b \quad \mid a c c i c o$} & 15 & & \\
\hline & Casos & Tasa & Casos & $\%^{\ddagger}$ & Tasa & Casos & $\%^{\ddagger}$ & $\% \%^{5}$ & Tasa & Casos & $\%^{\ddagger}$ & $\%$ & Tasa & Casos & $\%^{\ddagger}$ & $\% \%^{5}$ & Tasa \\
\hline 1991 & 801 & 24.1 & 371 & 46.3 & 3.0 & 330 & 41.2 & 88.9 & 34.7 & 16 & 2.0 & 4.3 & 9.4 & 25 & 3.1 & 6.7 & 5.6 \\
\hline 1992 & 517 & 13.4 & 329 & 63.6 & 3.3 & 271 & 52.4 & 82.4 & 21.2 & 26 & 5.0 & 7.9 & 14.8 & 32 & 6.2 & 9.7 & 7.0 \\
\hline 1993 & 684 & 16.1 & 459 & 67.1 & 2.9 & 404 & 59.1 & 88.0 & 24.2 & 18 & 2.6 & 3.9 & 10.0 & 37 & 5.4 & 8.1 & 7.5 \\
\hline 1994* & 765 & 17.0 & 518 & 67.7 & 2.8 & 428 & 55.9 & 82.6 & 27.2 & 12 & 1.6 & 2.3 & 6.0 & 78 & 10.2 & 15.1 & 12.5 \\
\hline 1995* & 884 & 16.7 & 571 & 64.6 & 2.1 & 466 & 52.7 & 81.6 & 21.4 & 11 & 1.2 & 1.9 & 7.1 & 94 & 10.6 & 16.5 & 13.6 \\
\hline 1996* & 847 & 14.2 & 502 & 59.3 & 1.6 & 396 & 46.8 & 78.9 & 15.8 & 5 & 0.6 & 1.0 & 2.5 & 101 & 11.9 & 20.1 & 10.5 \\
\hline
\end{tabular}

* Incluye información de hospitales e institutos nacionales

₹ Respecto al total de casos

$\S$ Respecto al total de población abierta

Tasa por 100 egresos hospitalarios

Fuente: Boletín de Información Estadística. D años a la salud, 1991-1996. Dirección General de Estadística e Informática, Secretaría de Salud

\section{Cuadro VII \\ MoRTALIDAD HOSPITALARIA POR DEFICIENCIAS DE LA NUTRICIÓN, POBLACIÓN DERECHOHABIENTE Estados Unidos Mexicanos, 1991-1996}

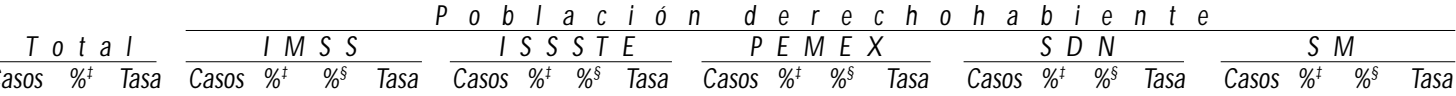

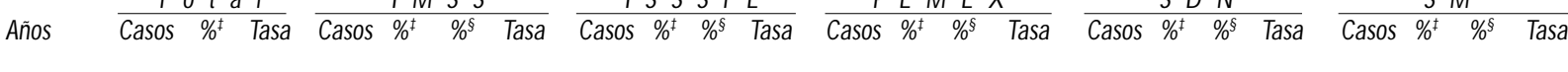

\begin{tabular}{lrrrrrrrrrrrrrrrrrrrrrrr}
1991 & 430 & 53.7 & 24 & 376 & 47 & 87.4 & 25.7 & 41 & 5.1 & 10 & 25.0 & 12 & 1 & 2.8 & 21.8 & 1 & 0.1 & 0 & 2.6 & 0 & 0.0 & 0.0 & 0.0 \\
\hline 1992 & 188 & 36.4 & 10 & 148 & 29 & 78.7 & 8.6 & 31 & 6.0 & 16 & 19.5 & 8 & 2 & 4.3 & 18.6 & 1 & 0.2 & 1 & 9.1 & 0 & 0.0 & 0.0 & 0.0 \\
\hline 1993 & 225 & 32.9 & 12 & 167 & 24 & 74.2 & 10.0 & 53 & 7.7 & 24 & 32.5 & 1 & 0 & 0.4 & 20.0 & 3 & 0.4 & 1 & 12.0 & 1 & 0.1 & 0.4 & 1.8 \\
\hline $1994^{*}$ & 247 & 32.3 & 12 & 191 & 25 & 77.3 & 10.6 & 46 & 6.0 & 19 & 25.7 & 7 & 1 & 2.8 & 14.6 & 3 & 0.4 & 1 & 5.0 & 0 & 0.0 & 0.0 & 0.0 \\
\hline $1995^{*}$ & 313 & 35.4 & 14 & 231 & 26 & 73.8 & 12.0 & 64 & 7.2 & 20 & 30.6 & 6 & 1 & 1.9 & 14.0 & 10 & 1.1 & 3 & 15.2 & 2 & 0.2 & 0.6 & 9.1 \\
\hline $1996_{*}^{*}$ & 345 & 40.7 & 15 & 258 & 30 & 74.8 & 13.1 & 68 & 8.0 & 20 & 30.9 & 10 & 1 & 2.9 & 20.4 & 9 & 1.1 & 3 & 13.4 & 0 & 0.0 & 0.0 & 0.0
\end{tabular} \\ * Incluye información de hospitales e institutos nacionales \\ ₹ Respecto al total de casos \\ $\S$ Respecto al total de población abierta \\ Tasa por 100 egresos hospitalarios \\ IMSS= Instituto Mexicano del Seguro Social \\ ISSSTE= Instituto de Seguridad y Servicios Sociales de los Trabajadores del Estado \\ PEMEX = Petróleos Mexicanos \\ SD N = Secretaría de la D efensa N acional \\ SM = Secretaría de Marina \\ Fuente: Boletín de Información Estadística. D años a la salud, 1991-1996. Dirección General de Estadística e Informática, Secretaría de Salud
}




\section{Cuadro VIII \\ MORBILIDAD HOSPITALARIA POR DEFICIENCIAS DE LA NUTRICIÓN, POBLACIÓN ABIERTA Estados Unidos Mexicanos, 1991-1996}

\begin{tabular}{|c|c|c|c|c|c|c|c|c|c|c|c|c|c|}
\hline \multirow[b]{2}{*}{ Años } & \multicolumn{2}{|c|}{ Tot a l } & To & & & $\begin{array}{ll}0 \mathrm{~b} \\
\mathrm{SA}\end{array}$ & $a \quad c$ & \multicolumn{3}{|c|}{$\begin{array}{llllll}b & i & e & r & t & a \\
D & D & F & & & \end{array}$} & \multicolumn{3}{|c|}{ IMSS-SOL } \\
\hline & Casos & Tasa & Casos & $\%^{\ddagger}$ & Casos & $\%^{\ddagger}$ & $\% \$$ & Casos & $\%^{\ddagger}$ & $\%$ & Casos & $\%^{\ddagger}$ & $\%^{5}$ \\
\hline 1991 & 3326 & 3.7 & 1564.0 & 47.0 & 950 & 28.6 & 60.7 & 170.0 & 5.1 & 10.9 & 444 & 13.3 & 28.4 \\
\hline 1992 & 3869 & 4.3 & 1910.0 & 49.4 & 1280 & 33.1 & 67.0 & 176.0 & 4.5 & 9.2 & 454 & 11.7 & 23.8 \\
\hline 1993 & 4261 & 4.8 & 2344.0 & 55.0 & 1672 & 39.2 & 71.3 & 180.0 & 4.2 & 7.7 & 492 & 11.5 & 21.0 \\
\hline 1994* & 4512 & 5.1 & 2399.0 & 53.2 & 1574 & 34.9 & 65.6 & 199.0 & 4.4 & 8.3 & 626 & 13.9 & 26.1 \\
\hline 1995* & 5285 & 6.0 & 3020.0 & 57.1 & 2174 & 41.1 & 72.0 & 155.0 & 2.9 & 5.1 & 691 & 13.1 & 22.9 \\
\hline 1996* & 5973 & 6.3 & 3659.0 & 61.3 & 2499 & 41.8 & 68.3 & 201.0 & 3.4 & 5.5 & 959 & 16.1 & 26.2 \\
\hline
\end{tabular}

* Incluye información de hospitales e institutos nacionales

₹ Respecto al total de casos

$\S$ Respecto al total de población abierta

Fuente: Boletín de Información Estadística. Daños a la salud, 1991-1996. Dirección General de Estadística e Informática, Secretaría de Salud

\section{MoRBILIDAd HOSPITALARIA POR DEFICIENCIAS DE LA NUTRICIÓN, POBLACIÓN DERECHOHABIENTE Estados Unidos Mexicanos, 1991-1996}

\begin{tabular}{|c|c|c|c|c|c|c|c|c|c|c|c|c|c|c|c|c|c|}
\hline \multirow[b]{2}{*}{ Años } & \multicolumn{2}{|c|}{ Tot a l } & & M S & & $\begin{aligned} 0 \quad b \\
15\end{aligned}$ & 51 & & $\begin{array}{c}d \\
P\end{array}$ & M & $x$ & $\mathrm{~h}$ a & $\begin{array}{ll}1 & \mathrm{e} \\
\mathrm{DNN} & \end{array}$ & l & & $5 \mathrm{M}$ & \\
\hline & Casos & $\%^{\ddagger}$ & $\overline{\text { Casos }}$ & $\%^{\ddagger}$ & $\% \$$ & Casos & $\%^{\ddagger}$ & $\%$ & Casos & $\%^{\ddagger}$ & $\%$ & $\overline{\text { Casos }}$ & $\%^{\ddagger}$ & $\% \%^{\S}$ & Casos & $\%^{\ddagger}$ & $\% \$$ \\
\hline 1991 & 1762 & 53.0 & 1465 & 44.0 & 83.1 & 164 & 4.9 & 9 & 55.0 & 2 & 3.1 & 39 & 1.2 & 2.2 & 39 & 1.2 & 2.2 \\
\hline 1992 & 1959 & 50.6 & 1716 & 44.4 & 87.6 & 159 & 4.1 & 8 & 43.0 & 1 & 2.2 & 11 & 0.3 & 0.6 & 30 & 0.8 & 1.5 \\
\hline 1993 & 1917 & 45.0 & 1668 & 39.1 & 87.0 & 163 & 3.8 & 9 & 5.0 & 0 & 0.3 & 25 & 0.6 & 1.3 & 56 & 1.3 & 2.9 \\
\hline 1994* & 2113 & 46.8 & 1799 & 39.9 & 85.1 & 179 & 4.0 & 8 & 48.0 & 1 & 2.3 & 60 & 1.3 & 2.8 & 27 & 0.6 & 1.3 \\
\hline 1995* & 2265 & 42.9 & 1925 & 36.4 & 85.0 & 209 & 4.0 & 9 & 43.0 & 1 & 1.9 & 66 & 1.2 & 2.9 & 22 & 0.4 & 1.0 \\
\hline 1996* & 2314 & 38.7 & 1962 & 32.8 & 84.8 & 220 & 3.7 & 10 & 49.0 & 1 & 2.1 & 67 & 1.1 & 2.9 & 16 & 0.3 & 0.7 \\
\hline
\end{tabular}

* Incluye información de hospitales e institutos nacionales

₹ Respecto al total de casos

§ Respecto al total de población derechohabiente

Tasa por 100 egresos hospitalarios

IMSS= Instituto Mexicano del Seguro Social

ISSSTE= Instituto de Seguridad y Servicios Sociales de los Trabajadores del Estado

PEMEX = Petróleos Mexicanos

SD N = Secretaría de la D efensa $N$ acional

SM = Secretaría de Marina

Fuente: Boletín de Información Estadística. D años a la salud, 1991-1996. Dirección General de Estadística e Informática, Secretaría de Salud 


\section{Consulta en MENORES de CINCO AÑOS SEgún ESTADo NUTRICIONAL \\ Estados Unidos Mexicanos, 1990-1996}

\begin{tabular}{|c|c|c|c|c|c|c|c|c|c|c|c|c|c|}
\hline \multirow[b]{3}{*}{ Año } & \multirow[b]{3}{*}{ Total } & \multirow{2}{*}{\multicolumn{2}{|c|}{ Sin desnutrición }} & \multicolumn{8}{|c|}{ Con desnutrición } & \multirow{2}{*}{\multicolumn{2}{|c|}{$\begin{array}{l}\text { Desnutridos } \\
\text { recuperados }\end{array}$}} \\
\hline & & & & \multicolumn{2}{|c|}{ Total } & \multicolumn{2}{|c|}{ Leve } & \multicolumn{2}{|c|}{ Moderada } & \multicolumn{2}{|c|}{ Severa } & & \\
\hline & & No. & $\%$ & No. & $\%$ & No. & $\%$ & No. & $\%$ & No. & $\%$ & No. & $\%$ \\
\hline 1990 & 5542077 & 4652525 & 83.95 & 839472 & 15.15 & 692546 & 12.50 & 128898 & 2.33 & 18028 & 0.33 & 50080 & 0.90 \\
\hline 1991 & 5895473 & 5063715 & 85.89 & 788096 & 13.37 & 658026 & 11.16 & 114984 & 1.95 & 15086 & 0.26 & 43662 & 0.74 \\
\hline 1992 & 5714429 & 5129523 & 89.76 & 562706 & 9.85 & 481053 & 8.42 & 72947 & 1.28 & 8706 & 0.15 & 22200 & 0.39 \\
\hline 1993 & 6089737 & 5544194 & 91.04 & 527505 & 8.66 & 455635 & 7.48 & 64130 & 1.05 & 7740 & 0.13 & 18038 & 0.30 \\
\hline 1994 & 6189627 & 5660732 & 91.46 & 511072 & 8.26 & 439088 & 7.09 & 63678 & 1.03 & 8306 & 0.13 & 17823 & 0.29 \\
\hline 1995 & 7073728 & 6473430 & 91.51 & 581374 & 8.22 & 488954 & 6.91 & 79698 & 1.13 & 12722 & 0.18 & 18924 & 0.27 \\
\hline 1996 & 7293963 & 6571187 & 90.09 & 695410 & 9.53 & 573449 & 7.86 & 106450 & 1.46 & 15511 & 0.21 & 27366 & 0.38 \\
\hline
\end{tabular}

Fuente: Sistema de Información en Salud para Población A bierta, Subsistema de Servicios, Dirección General de Estadística e Informática, Secretaría de Salud 


\section{Cuadro XI \\ Total de CONSULtas en Menores de Cinco años SEgún ESTAdo NUtricional Estados Unidos Mexicanos 1996}

\begin{tabular}{|c|c|c|c|c|c|c|c|c|c|c|c|c|c|}
\hline \multirow[b]{3}{*}{ Entidad federativa } & \multirow[b]{3}{*}{ Total } & \multirow{2}{*}{\multicolumn{2}{|c|}{ Sin desnutrición }} & & \multirow{2}{*}{\multicolumn{6}{|c|}{$d$ es nutrición }} & \multirow{2}{*}{\multicolumn{2}{|c|}{$\begin{array}{l}\text { Desnutridos } \\
\text { recuperados }\end{array}$}} \\
\hline & & & & \multicolumn{2}{|c|}{ Total } & \multicolumn{2}{|c|}{ Leve } & & & & Severa & & \\
\hline & & No. & $\%$ & No. & $\%$ & No. & $\%$ & No. & $\%$ & No. & $\%$ & No. & \\
\hline Estados Unidos Mexicanos & 7293963 & 6571187 & 90.09 & 695410 & 9.53 & 573449 & 7.86 & 106450 & 1.46 & 15511 & 0.21 & 27366 & 0.38 \\
\hline Aguascalientes & 83088 & 68989 & 83.03 & 6970 & 8.39 & 5874 & 7.07 & 940 & 1.13 & 156 & 0.19 & 159 & \\
\hline Baja California & 60933 & 57518 & 94.40 & 1686 & 2.77 & 1343 & 2.20 & 245 & 0.40 & 98 & 0.16 & 43 & 0.07 \\
\hline Baja C alifornia Sur & 45410 & 42018 & 92.53 & 1663 & 3.66 & 1441 & 3.17 & 205 & 0.45 & 17 & 0.04 & 66 & 0.15 \\
\hline Campeche & 87405 & 62962 & 72.03 & 12105 & 13.85 & 9703 & 11.10 & 2146 & 2.46 & 256 & 0.29 & 233 & 0.27 \\
\hline Coahuila & 126573 & 110463 & 87.27 & 7824 & 6.18 & 6467 & 5.11 & 1252 & 0.99 & 105 & 0.08 & 462 & 0.3 \\
\hline Colima & 70263 & 63092 & 89.79 & 3456 & 4.92 & 2911 & 4.14 & 486 & 0.69 & 59 & 0.08 & 259 & 0.3 \\
\hline Chiapas & 225316 & 159064 & 70.60 & 32354 & 14.36 & 25822 & 11.46 & 5636 & 2.50 & 896 & 0.40 & 1544 & 0.69 \\
\hline Chihuahua & 93973 & 80022 & 85.15 & 6684 & 7.11 & 4879 & 5.19 & 1411 & 1.50 & 394 & 0.42 & 583 & 0.62 \\
\hline Distrito Federal & 435887 & 382399 & 87.73 & 26604 & 6.10 & 23726 & 5.44 & 2770 & 0.64 & 108 & 0.02 & 280 & 0.06 \\
\hline Durango & 78182 & 69099 & 88.38 & 4324 & 5.53 & 3730 & 4.77 & 538 & 0.69 & 56 & 0.07 & 435 & 0.56 \\
\hline Guanajuato & 397310 & 326343 & 82.14 & 35060 & 8.82 & 29434 & 7.41 & 4990 & 1.26 & 636 & 0.16 & 847 & 0.2 \\
\hline Guerrero & 386643 & 274537 & 71.01 & 55330 & 14.31 & 45719 & 11.82 & 8598 & 2.22 & 1013 & 0.26 & 1446 & 0.3 \\
\hline Hidalgo & 223903 & 178310 & 79.64 & 22246 & 9.94 & 19080 & 8.52 & 2865 & 1.28 & 301 & 0.13 & 1101 & 0.4 \\
\hline Jalisco & 515975 & 427358 & 82.83 & 42797 & 8.29 & 35967 & 6.97 & 5440 & 1.05 & 1390 & 0.27 & 3023 & 0.59 \\
\hline México & 1723841 & 1480525 & 85.89 & 120340 & 6.98 & 99888 & 5.79 & 18700 & 1.08 & 1752 & 0.10 & 2636 & 0.15 \\
\hline Michoacán & 157087 & 132838 & 84.56 & 11800 & 7.51 & 10130 & 6.45 & 1364 & 0.87 & 306 & 0.19 & 649 & 0.4 \\
\hline Morelos & 168283 & 130617 & 77.62 & 18376 & 10.92 & 15279 & 9.08 & 2661 & 1.58 & 436 & 0.26 & 914 & 0.54 \\
\hline N ayarit & 77606 & 67595 & 87.10 & 4811 & 6.20 & 3995 & 5.15 & 668 & 0.86 & 148 & 0.19 & 389 & 0.50 \\
\hline Nuevo León & 339532 & 326100 & 96.04 & 6463 & 1.90 & 5496 & 1.62 & 815 & 0.24 & 152 & 0.04 & 506 & 0.15 \\
\hline 0 axaca & 314655 & 203077 & 64.54 & 54897 & 17.45 & 45484 & 14.46 & 8393 & 2.67 & 1020 & 0.32 & 1784 & 0.5 \\
\hline Puebla & 6759 & 230608 & 75.18 & 37232 & 12.14 & 30437 & 9.92 & 5840 & 1.90 & 955 & 0.31 & 1687 & 0.5 \\
\hline Q uerétaro & 105472 & 84263 & 79.89 & 10363 & 9.83 & 8458 & 8.02 & 1671 & 1.58 & 234 & 0.22 & 483 & 0.46 \\
\hline Q uintana Roo & 65750 & 44731 & 68.03 & 10298 & 15.66 & 8380 & 12.75 & 1632 & 2.48 & 286 & 0.43 & 423 & 0.64 \\
\hline San Luis Potosí & 138998 & 95499 & 68.71 & 21436 & 15.42 & 15944 & 11.47 & 4486 & 3.23 & 1006 & 0.72 & 627 & 0.45 \\
\hline Sinaloa & 136768 & 127264 & 93.05 & 4541 & 3.32 & 3638 & 2.66 & 793 & 0.58 & 110 & 0.08 & 422 & 0.31 \\
\hline Sonora & 193550 & 185515 & 95.85 & 3841 & 1.98 & 3242 & 1.68 & 528 & 0.27 & 71 & 0.04 & 353 & 0.18 \\
\hline Tabasco & 540308 & 478005 & 88.47 & 30379 & 5.62 & 23877 & 4.42 & 5442 & 1.01 & 1060 & 0.20 & 1545 & 0.29 \\
\hline Tamaulipas & 106542 & 94634 & 88.82 & 5837 & 5.48 & 5106 & 4.79 & 645 & 0.61 & 86 & 0.08 & 234 & 0.22 \\
\hline Tlaxcala & 207378 & 161838 & 78.04 & 22096 & 10.65 & 18677 & 9.01 & 3012 & 1.45 & 407 & 0.20 & 1348 & 0.65 \\
\hline Veracruz & 319086 & 246332 & 77.20 & 35517 & 11.13 & 29060 & 9.11 & 5375 & 1.68 & 1082 & 0.34 & 1720 & 0.54 \\
\hline Yucatán & 142178 & 86592 & 60.90 & 27517 & 19.35 & 21310 & 14.99 & 5467 & 3.85 & 740 & 0.52 & 552 & 0.33 \\
\hline Zacatecas & 114719 & 92980 & 81.05 & 10563 & 9.21 & 8952 & 7.80 & 1436 & 1.25 & 175 & 0.15 & 613 & \\
\hline
\end{tabular}

Fuente: Sistema de Información en Salud para Población Abierta, Subsistema de Servicios, Dirección General de Estadística e Informática, Secretaría de Salud 


\section{Cuadro XII \\ Distribución de los niños de PRimer grado de PRIMARIA SEgún PReVAlencia de déficit de talla Estados Unidos MeXICANOS 1993-1994}

Entidad federativa

Estados Unidos Mexicanos

Aguascalientes

Baja California

Baja California Sur

Campeche

Coahuila

Colima

Chiapas

Chihuahua

Distrito Federal

Durango

Guanajuato

Guerrero

Hidalgo

Jalisco

México

Michoacán

Morelos

$\mathrm{N}$ ayarit

Nuevo León

0 axaca

Puebla

Q uerétaro

Q uintana Roo

San Luis Potosí

Sinaloa

Sonora

Tabasco

Tamaulipas

Tlaxcala

Veracruz

Yucatán

Zacatecas

Punto de corte: $-2.00 \mathrm{DE}$ de la talla para la edad

Fuente: *Primer Censo N acional de Talla en N iños de Primer Grado de Primaria, Secretaría de Educación Pública/Sistema para el Desarrollo Integral de la Familia

₹ Segundo Censo N acional de Talla en N iños de Primer Grado de Primaria, Secretaría de Educación Pública/Sistema para el Desarrollo Integral de la Familia

Niños Prevalencia

$(\%)$

2589577

18.4

2344132

18.7

23604

48025

8888

20221

54721

12440

131711

77401

179020

41608

149151

113653

62520

180179

294966

111866

37237

27275

79009

113792

138166

37442

17443

67410

70066

55685

55270

57978

25392

207827

50875

38736

7.4

23790

95536

18426

51131

13532

119222

64656

172196

39412

125588

87226

61940

154186

289041

83782

35284

24816

68431

104604

127299

36697

18367

61396

57089

51102

55790

55387

21915

182864

48424

41448

Prevalencia

(\%)

7.5

4.8

4.3

30.1

6.2

9.0

44.1

7.8

1993 *

1994 末 